\title{
Fenóis totais, atividade antioxidante e constituintes químicos de extratos de Anacardium occidentale L., Anacardiaceae
}

\author{
Mariana H. Chaves*, Antônia Maria das Graças Lopes Citó, José Arimatéia Dantas Lopes, \\ Danielly Albuquerque da Costa, Carlos Antônio A. de Oliveira, Ana Flávia Costa e Francisco \\ Eleodoro M. Brito Júnior
}

\author{
Centro de Ciências da Natureza, Departamento de Química, Universidade Federal do Piauí, 64049-550 \\ Teresina-PI, Brasil.
}

\begin{abstract}
RESUMO: O presente artigo relata o isolamento e identificação do palmitato, oleato e linoleato de sitosterila, sitosterol, estigmasterol, 3-O- $\beta$-D-galactopiranosídeo do sitosterol, 3-O- $\beta$-Dgalactopiranosídeo do estigmasterol, 3-O- $\beta$-D-glicopiranosídeo do sitosterol e uma mistura de ácidos anacárdicos (monoeno e dieno) do extrato etanólico de cascas do caule de Anacardium occidentale L., Anacardiaceae, bem como do sitosterol, estigmasterol, lupeol, $\beta$-amirina, catequina e epicatequina do extrato etanólico do tegumento da castanha de caju in natura. Os extratos EtOH da casca e do tegumento foram avaliados quanto ao conteúdo de fenóis totais e atividade antioxidante. O extrato etanólico das cascas do caule apresentou maior conteúdo de compostos fenólicos e percentual de atividade antioxidante.
\end{abstract}

Unitermos: Anacardium occidentale, catequinas, esteroides, fenóis totais, atividade antioxidante.

\begin{abstract}
Total phenolics, antioxidant activity and chemical constituents from extracts of Anacardium occidentale L., Anacardiaceae". This paper describes the isolation and identification of a mixture of sitosteryl ester derivatives of fatty acids (palmitic, oleic and linoleic), sitosterol, stigmasterol, sitosterol-3-O- $\beta$-galactopyranoside, stigmasterol-3- $O$ - $\beta$-galactopyranoside, sitosterol3-O- $\beta$-glucopyranoside and a mixture of anacardic acids (monoene and diene) from stem bark of Anacardium occidentale L., Anacardiaceae, as well as sitosterol, stigmasterol, lupeol, $\beta$-amyrin, catechin and epicatechin from in natura cashew nut testa. Ethanol extracts from stem bark and testa were analyzed for antioxidant activity and total phenol content. The ethanol extract from stem bark exhibited the maximum of antioxidant activity and phenol content.
\end{abstract}

Keywords: Anacardium occidentale, catequins, esteroids, total phenol, antioxidant activity.

\section{INTRODUÇÃO}

Anacardium occidentale L., Anacardiaceae, conhecida popularmente como cajueiro, é uma planta nativa do Brasil e característica dos campos e das regiões costeiras do Norte e Nordeste. Foi introduzida na África e Índia, pelos portugueses no século XVI e hoje está disseminada por todas as regiões tropicais do globo (Pio Corrêa, 1926). A Índia é atualmente o principal produtor e exportador mundial da castanha-de-caju, seguido pelo Vietnã e Brasil. Os Estados brasileiros que se destacam são o Ceará, como maior produtor, seguido pelo Rio Grande do Norte e Piauí (Moura, 2007).

$\mathrm{O}$ cajueiro é uma árvore de grande porte em bons terrenos, atingindo até $20 \mathrm{~m}$ de altura. Em terras secas e arenosas do sertão torna-se uma árvore baixa, esgalhada $\mathrm{e}$ às vezes quase rasteira. A casca é adstringente, com ação antidiabética e usada popularmente em loções e gargarejos contra aftas e infecções da garganta, dela exsuda por incisão uma goma-resina amarela e dura com propriedades depurativa e expectorante. $\mathrm{O}$ suco das folhas novas é antiescorbútico e eficiente para combater aftas e cólicas intestinais. As flores, muito visitadas pelas abelhas são tônicas e até afrodisíacas (Pio Corrêa, 1926; Agra et al., 2007). A raiz é purgativa e os frutos ou cotilédones, seu principal produto, conhecido como castanha de caju ou noz de caju são comestíveis depois de assados e muito apreciados no exterior como tira-gosto ou na manufatura de doces. Em alguns países a castanha é consumida até mesmo crua e exerce uma grande competitividade com a produção de nozes, amendoins, avelãs, pistaches, entre outras. A elas são atribuídas propriedades tônico-excitantes, úteis contra a impotência e na debilidade decorrente de grandes enfermidades (Pio Corrêa, 1926; Agra et al., 2007).

Depois da castanha, o segundo produto principal do cajueiro é o LCC (líquido da casca da castanha de caju) conhecido internacionalmente por CNSL (cashew nutshell liquid), seguido pelo suco da polpa do pseudofruto ou 
pedúnculo, denominado caju (Mothé \& Milfont Jr, 1994).

O LCC é viscoso, castanho escuro, acre e caústico, que impregna o mesocarpo da castanha sendo liberado no processamento para obtenção da amêndoa, constitui uma fonte natural de compostos fenólicos e sua composição química é bem estabelecida. Seus constituintes são ácidos anacárdicos, cardóis, cardanóis e 2-metilcardóis. Estes compostos apresentam uma cadeia lateral alifática de quinze carbonos na posição meta em relação à hidroxila, contendo de zero a três insaturações localizadas em C-8' (monoeno), C-8' e C-11' (dieno) e C-8', C-11' e C-14' (trieno) (Tyman et al., 1989; Kubo et al., 1986; Shobba et al., 1992).

A composição do LCC varia de acordo com sua origem e também com o processo de extração (Gedam \& Sampathkumaran, 1986). Se a extração é realizada a temperaturas entre $180-200^{\circ} \mathrm{C}$, como adotada no processo industrial, resulta na descarboxilação do ácido anacárdico, conduzindo ao cardanol (Tychopoulos \& Tyman, 1990). A Tabela 1 mostra a variação da composição dos constituintes fenólicos do LCC, de acordo com a cadeia lateral, obtida por diversas técnicas (Gedam \& Sampathkumaran, 1986).

Tabela 1. Variação da composição dos constituintes fenólicos do LCC (Gedam \& Sampathkumaran, 1986)

\begin{tabular}{ccccc}
\hline Constituintes & $\begin{array}{c}\text { Ácido } \\
\text { anacárdico } \\
(\%)\end{array}$ & $\begin{array}{c}\text { Cardanol } \\
(\%)\end{array}$ & $\begin{array}{c}\text { Cardol } \\
(\%)\end{array}$ & $\begin{array}{c}\text { 2-metil- } \\
\text { cardol (\%) }\end{array}$ \\
\hline $\mathrm{C} 15: 0$ & $2,2-3,0$ & $3,9-4,4$ & $0,2-2,7$ & $0,9-1,3$ \\
$\mathrm{C} 15: 1$ & $25,0-33,3$ & $21,6-32,2$ & $8,4-15,2$ & $16,3-25,3$ \\
$\mathrm{C} 15: 2$ & $17,8-32,1$ & $15,4-18,2$ & $24,2-28,9$ & $20,6-24,4$ \\
$\mathrm{C} 15: 3$ & $36,3-50,4$ & $45,2-59,0$ & $36,5-67,2$ & $49,8-62,2$ \\
\hline
\end{tabular}

O óleo da amêndoa (46,5\%, em média) apresenta em sua composição, os ácidos: palmítico, C16:0 (11,6\%), palmitoléico, C16:1 (0,3\%), heptadecanóico, C17:0 (0,1\%), esteárico, C18:0 (8,9\%), oléico, C18:1 (61,5\%), linoléico, C18:2 (17,1\%), araquídico, C20:0 (0,5\%) e linolênico, C18:3 (traços), determinados como ésteres metílicos, por cromatografia gasosa (Toschi et al., 1993). Além dos triacilgliceróis, componentes majoritários, o óleo contém lipídios fenólicos (derivados de ácidos anacárdicos, cardóis e 2-metilcardóis), tocoferóis ( $\alpha, \gamma$ e $\delta)$ (Kubo et al., 1986; Shobba et al., 1992; Toschi et al., 1993) e outros constituintes menos abundantes, determinados na fração insaponificável do óleo $(1,4 \%)$, tais como: esteroides (colesterol, campesterol, 24-metilenocolesterol, estigmasterol, sitosterol, fucosterol e $\Delta^{5}$-avenasterol), 4-metil-esteroides (obtusifoliol, diidroobtusifoliol, ciclolaudenol, gramiesterol e citrostadienol) e triterpenos (cicloartanol, $\beta$-amirina, cicloartenol, ciclolaudenol e 24metilenocicloartanol) (Toschi et al., 1993).

Além dos lipídios fenólicos destacam-se outros constituintes químicos isolados e identificados nas diversas partes do cajueiro como os flavonóides apigenina, kanferol, quercetina, quercetina-3-O-glicopiranosila, quercetina-
3-O-ranminopiranosila, miricetina, agatisflavona, robustoflavona e amentoflavona nas folhas (Arya et al., 1989); naringenina, naringenina-7-O-(6"-O-p-cumaroil)$\beta$-D-glicosila (Rahman et al., 1978), naringenina-5 $\beta$ glicosila (Murthy et al., 1982) occidentosideo, também conhecido como (-)-salipurposideo (Murthy et al., 1981; Murthy et al., 1982) nas cascas da castanha; (+)-catequina e (-)-epicatequina no tegumento (Subrahmanian \& Nair, 1969). Além destes foram isolados galato de etila das flores (Subrahmanian et al., 1969); sitosterol das cascas da castanha (Murthy et al., 1982); ácido gálico, como produto de hidrólise de taninos, myoinositol, colesterol, campesterol, estigmasterol e sitosterol das cascas do caule (Mota, 1982; Dinda et al., 1987).

Diversas atividades farmacológicas têm sido testadas em extratos de $A$. occidentale, porém atenção especial foi dada aos lipídios fenólicos, sobretudo por suas propriedades antioxidantes (Kubo et al., 2006; Kamath \& Rajini, 2007). A cadeia alquílica presente nestes compostos exercem influência significativa na atividade biológica, podendo está relacionada com o aumento da solubilidade das porções fenólicas a qual é responsável pela proteção contra a degradação biológica ou oxidação química. A influência da cadeia alquílica no mecanismo de proteção é semelhante ao efeito proposto para a cadeia isoprênilica do tocoferol (Correia et al., 2006). O ácido anacárdico exibe propriedades moluscicida (Kubo et al., 1986), antitumoral (Itokawa et al., 1987; Kubo et al., 1993), antimicrobiana (Himejima \& Kubo, 1991), inibidor de prostaglandina sintase (Kubo et al, 1987) e lipooxigenase (Grazzini et al., 1991).

O presente trabalho teve como objetivo avaliar a atividade antioxidante, determinar os fenóis totais, isolar e identificar constituintes químicos de extratos etanólicos da casca do caule e do tegumento da castanha de caju in natura.

\section{MATERIAL E MÉTODOS}

\section{Métodos gerais}

As separações cromatográficas em coluna à pressão atmosférica, foram realizadas em gel de sílica 60 (0,063-0,20 mm) da Merck e Sephadex LH-20 da Sigma. As placas para cromatografia em camada delgada analítica (CCDA) foram preparadas com $0,25 \mathrm{~mm}$ de espessura, usando como fase estacionária, gel de sílica $60 \mathrm{GF}_{254}$ da Merck e $60 \mathrm{G}$ Vetec. As revelações das cromatoplacas foram feitas por borrifamento com solução de sulfato cérico. O reagente Folin-Ciocalteu foi adquirido da Merck, o radical DPPH (2,2-difenil-1-picril-hidrazila) da Aldrich Co e o ácido gálico da Vetec.

Os espectros de ressonância magnética nuclear (RMN) foram obtidos em espectrômetro Brüker, modelo Avance DRX-500, operando a $500 \mathrm{MHz}$ na frequência do hidrogênio e a $125 \mathrm{MHz}$ na frequência do carbono- 
13. O sinal do solvente residual, não deuterado, ou do TMS foi usado como referência interna. Os solventes utilizados na dissolução das amostras para obtenção dos espectros foram $\mathrm{CDCl}_{3}$ e $\mathrm{CD}_{3} \mathrm{OD}$ (Isotec-INC). As análises dos ésteres metílicos por CG/EM foram feitas com o uso de um cromatógrafo gasoso CG 17-A com detector de massas Shimadzu, modelo GC/MS-QP 5050A, gerenciado pelo software Class-5000, usando-se coluna capilar de sílica fundida DB-5 (95\% de metilpolisiloxano e $5 \%$ de fenilpolisiloxano) de $50 \mathrm{~m}$ de comprimento, com $0,25 \mathrm{~mm}$ de diâmetro interno e $0,25 \mu \mathrm{m}$ de espessura do filme. As condições de operação foram as seguintes: temperatura programada da coluna de $100{ }^{\circ} \mathrm{C}$ por $1 \mathrm{~min}$, taxa de aquecimento a $4{ }^{\circ} \mathrm{C} / \mathrm{min}$ até $200^{\circ} \mathrm{C}$ permanecendo por $7 \mathrm{~min}$, taxa de aquecimento a $10{ }^{\circ} \mathrm{C} / \mathrm{min}$ até $280^{\circ} \mathrm{C}$ permanecendo por $4 \mathrm{~min}$; a temperatura do injetor foi 200 ${ }^{\circ} \mathrm{C}$ e da interface, $250{ }^{\circ} \mathrm{C}$; o gás de arraste foi o hélio, com fluxo de $0,6 \mathrm{~mL} / \mathrm{min}$. A corrida com detector de massa foi feita no modo scan, com tempo de aquisição de $42: 50 \mathrm{~min}$ e corte do solvente em $5 \mathrm{~min}$; faixa de massas: 40 a $400 \mathrm{D}$. Os compostos foram identificados através de comparação com os espectros de massas da biblioteca Wiley229.

\section{Material vegetal}

Cascas do caule e castanha in natura de Anacardium occidentale L., Anacardiaceae, foram coletadas no Campus da Universidade Federal do Piauí nos meses de março e julho de 2003, respectivamente. Uma exsicata do espécimem coletado (TEPB 674) encontra-se depositada no Herbário Graziela Barroso, UFPI.

\section{Preparação e fracionamento do extrato da casca do caule}

As cascas foram secas ao ar e trituradas em moinho de facas fornecendo $718 \mathrm{~g}$ de pó, o qual foi submetido à extração com etanol por três vezes consecutivas à temperatura ambiente. $\mathrm{O}$ extrato etanólico foi parcialmente concentrado a um volume de $1032 \mathrm{~mL}$ e determinado o peso seco que correspondeu a $99 \mathrm{~g}(14 \% \mathrm{em}$ relação ao material vegetal seco). $\mathrm{O}$ extrato foi novamente concentrado até o volume de $200 \mathrm{~mL}$, adicionado $400 \mathrm{~mL}$ de água e submetido à partição com éter etílico (4x 200 $\mathrm{mL}$ ). A fase etérea foi concentrada, suspensa em $\mathrm{MeOH}-$ $\mathrm{H}_{2} \mathrm{O}$ (9:1) e extraída com hexano, fornecendo as fases hexânica $(2,3 \mathrm{~g})$ e hidroalcoólica $(2 \mathrm{~g})$. A fase hexânica foi submetida à cromatografia em coluna de Sephadex LH-20 eluída com hexano- $\mathrm{CH}_{2} \mathrm{Cl}_{2}$ 1:4 (frações 1-28), $\mathrm{CH}_{2} \mathrm{Cl}_{2}$-acetona 3:2 (frações 29-34), $\mathrm{CH}_{2} \mathrm{Cl}_{2}$-acetona 4:1 (frações 35-52) e MeOH (frações 53-55). As frações foram reunidas em dez grupos, após análise por cromatografia em camada delgada analítica (CCDA). O grupo A2 (1,45 $\mathrm{g}$, frações 2-13) foi novamente submetido à cromatografia em coluna de gel de sílica eluída com hexano-AcOEt em ordem crescente de polaridade fornecendo A2-7 (321 mg,
hexano-AcOEt 97:3), correspondente à mistura de ésteres de ácidos graxos e sitosterol $(\mathbf{1 a}+\mathbf{1 b}+\mathbf{1 c})$, e A2-25 (485 $\mathrm{mg}$, hexano-AcOEt 9:1), que depois de recristalizada em $\mathrm{MeOH}$ forneceu $400 \mathrm{mg}$ da mistura de sitosterol (2) e estimasterol (3). O grupo A21 (257 mg, frações 21-28) foi novamente submetido à cromatografia em coluna de gel de sílica, eluída com $\mathrm{CH}_{2} \mathrm{Cl}_{2}-\mathrm{MeOH}$ em ordem crescente de polaridade fornecendo $15 \mathrm{mg}$ da mistura dos esteroides sitosterol e estigmasterol galactosilados $(\mathbf{2} \mathbf{a}+\mathbf{3 a} \mathbf{a})$. Os grupos A30 (133 mg, frações 30-33) e A44 (40 mg, frações 4445) corresponderam à mistura de ácidos anacárdicos com cadeia insaturada $(\mathbf{4 a} \mathbf{a}+\mathbf{4 b})$ e ao 3-O- $\beta$-D-glicopiranosídeo do sitosterol (2b), respectivamente.

\section{Hidrólise dos ésteres de ácidos graxos e sitosterol $(1 a+1 b+1 c)$}

Uma alíquota de $32 \mathrm{mg}$ da mistura de ésteres de ácidos graxos e sitosterol (A2-7) foi refluxada por $1 \mathrm{~h}$, com solução de $\mathrm{KOH}$ (32 mg) em metanol (7,5 mL). Terminada a reação, cerca de $80 \%$ do metanol foi evaporado, restabelecido o volume inicial com água e extraído com éter etílico, fornecendo, após concentração do solvente, 24 $\mathrm{mg}$ de sitosterol. A fase aquosa foi tratada com $\mathrm{H}_{2} \mathrm{SO}_{4} 1 \%$ até $\mathrm{pH} 2$ e extraída com éter etílico. A fase etérea depois de concentrada foi tratada com excesso de solução etérea de diazometano, sob refrigeração, obtendo-se uma mistura de ésteres metílicos, os quais foram identificados por $\mathrm{CG} / \mathrm{EM}$ (Silva et al., 2007).

\section{Preparação e fracionamento do extrato do tegumento}

A castanha in natura foi coletada e separada em tegumento e amêndoa. O tegumento foi liofilizado $(90 \mathrm{~g})$ e extraído três vezes por maceração com etanol à temperatura ambiente. O extrato etanólico (19 g, 21\%) foi submetido à partição fornecendo as fases hexânica $(1,4 \mathrm{~g})$ e acetato de etila $(5,3 \mathrm{~g})$. A fase hexânica foi saponificada com solução metanólica de $\mathrm{KOH}(0,5 \% \mathrm{~m} / \mathrm{v})$, por $1 \mathrm{~h}$. Cerca de $90 \%$ do metanol foi removido da mistura reacional em evaporador rotativo e o volume inicial foi restabelecido com água e extraído com éter etílico (Chaves et al., 2004). A fase etérea contendo o material insaponificável (126 mg) foi aplicada em coluna cromatográfica de gel de sílica eluída com hexano-AcOEt 95:5 (frações 6-13), 9:1 (frações 14-36), 8:2 (frações 37-41) e AcOEt 100\% (frações 42-59). Após análise por CCDA, as frações foram reunidas em quatro grupos. Os grupos f6 (frações 6-10) e f14 (frações 14-19) consistiram das misturas de lupeol e $\beta$-amirina $(\mathbf{5}+\mathbf{6}, 15 \mathrm{mg})$ e sitosterol e estimasterol $(\mathbf{2}+\mathbf{3}$, $20 \mathrm{mg}$ ), respectivamente. Uma alíquota de $1,0 \mathrm{~g}$ da fase acetato de etila foi fracionada por duas vezes consecutivas em colunas de Sephadex LH-20, sendo a primeira eluída com $\mathrm{MeOH}-\mathrm{CHCl}_{3}$ (3:2) e a segunda com $\mathrm{MeOH} 100 \%$, resultando na mistura de catequina e epicatequina $(\mathbf{7 + 8}, 15$ $\mathrm{mg})$. 


\section{Determinação dos fenóis totais}

A determinação do teor de fenóis totais nos extratos EtOH do tegumento da castanha e de casca do caule foi feita por meio de espectroscopia na região do visível utilizando o método de Folin-Ciocalteu (Sousa et al., 2007). O teor de fenóis totais (FT) foi determinado por interpolação da absorvância das amostras contra uma curva de calibração construída com padrões de ácido gálico (10 a $350 \cdot \mathrm{g} / \mathrm{mL}$ ) e expressa como miligramas de EAG (equivalentes de acido gálico) por grama de extrato. A equação da curva de calibração do ácido gálico foi $C$ $=809,0200 A+5,0827$, com coeficiente de correlação $R=$ 0,999 , onde $C$ é a concentração do ácido gálico e $A$ é a absorvância a $750 \mathrm{~nm}$. Todas as análises foram realizadas em triplicata.

\section{Determinação da atividade antioxidante}

A avaliação quantitativa da atividade antioxidante foi feita monitorando o consumo do radical livre DPPH pelas amostras, por meio da medida do decréscimo da absorvância de soluções de diferentes concentrações em espectrofotômetro UV-Vis no comprimento de onda 516 nm, tendo como controle positivo o BHT. Soluções dos extratos EtOH $(500 \mu \mathrm{g} / \mathrm{mL})$ e do controle positivo em metanol foram diluídas nas concentrações de 250, 200, $150,100,50$ e $25 \mu \mathrm{g} / \mathrm{mL}$. As medidas das absorvâncias das misturas reacionais $(0,3 \mathrm{~mL}$ da solução da amostra ou do controle positivo e $2,7 \mathrm{~mL}$ da solução estoque de DPPH na concentração de $40 \mu \mathrm{g} / \mathrm{mL}$ ), foram feitas a 516 $\mathrm{nm}$, no primeiro, quinto e décimo minuto, a cada $10 \mathrm{~min}$ até completar $30 \mathrm{~min}$. A mistura de metanol $(2,7 \mathrm{~mL})$ e solução metanólica do extrato $(0,3 \mathrm{~mL})$ foi utilizada como branco.
Os valores de absorvância em todas as concentrações testadas, no tempo de $30 \mathrm{~min}$, foram convertidos em porcentagem de atividade antioxidante (AA), determinada pela seguinte equação:

$$
\% \mathrm{AA}=\left\{\left[\mathrm{Abs}_{\mathrm{DPPH}^{-}}-\left(\mathrm{Abs}_{\text {amostra }^{-}}-\mathrm{Abs}_{\text {branco }}\right)\right] \times 100\right\} / \mathrm{Abs}_{\mathrm{DPPH}}
$$

onde $\mathrm{Abs}_{\mathrm{DPPH}}$ é a absorvância inicial da solução metanólica de DPPH e Abs ${ }_{\text {amostra }}$ é a absorvância da mistura reacional (DPPH+amostra) (Sousa et al., 2007).

\section{RESULTADOS E DISCUSSÃO}

O fracionamento da fase hexânica da partição do extrato etanólico das cascas do caule de Anacardium occidentale L., Anacardiaceae, em coluna de Sephadex LH-20 resultou no isolamento de três misturas de esteroides, uma composta de palmitato, oleato e linoleato de sitosterila $(\mathbf{1} \mathbf{a}+\mathbf{1} \mathbf{b}+\mathbf{1 c})$, outra constituída por sitosterol (2) e estigmasterol (3), e uma terceira formada por 3-O$\beta$-D-galactopiranosídeo do sitosterol (2a) e 3-O- $\beta$-Dgalactopiranosídeo do estigmasterol (3a). Adicionalmente foi também isolada uma mistura de ácidos anacárdicos com cadeia insaturada $(\mathbf{4 a}+\mathbf{4 b})$ e o $3-O-\beta-\mathrm{D}$-glicopiranosídeo do sitosterol (2b). O extrato etanólico do tegumento, após partição com solventes, resultou na fase hexânica e acetato de etila. A fração insaponificável da fase hexânica depois de submetida à cromatografia em coluna de gel de sílica forneceu uma mistura dos triterpenoides lupeol (5) e $\beta$-amirina (6) e outra dos esteroides sitosterol e estigmasterol. O fracionamento da fase acetato de etila em coluna de Sephadex LH-20 resultou no isolamento da mistura de catequina (7) e epicatequina (8).

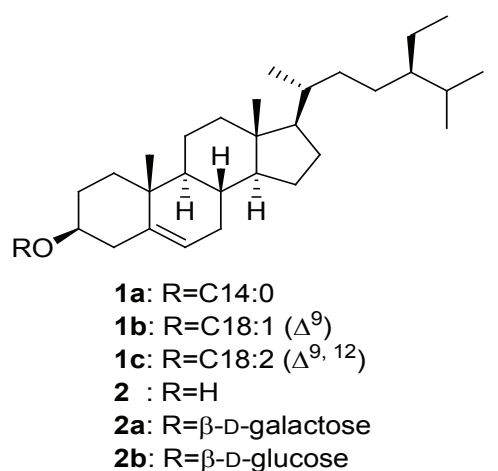<smiles>[R]c1cccc(O)c1C(=O)O</smiles>

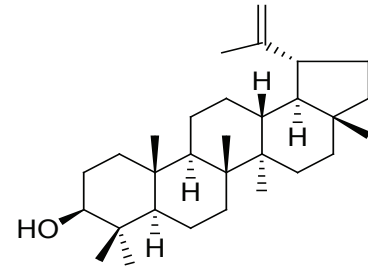

5

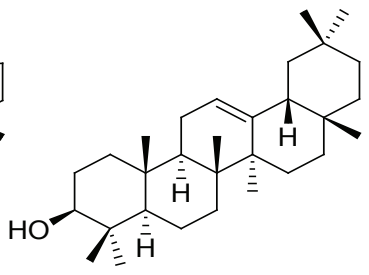

6
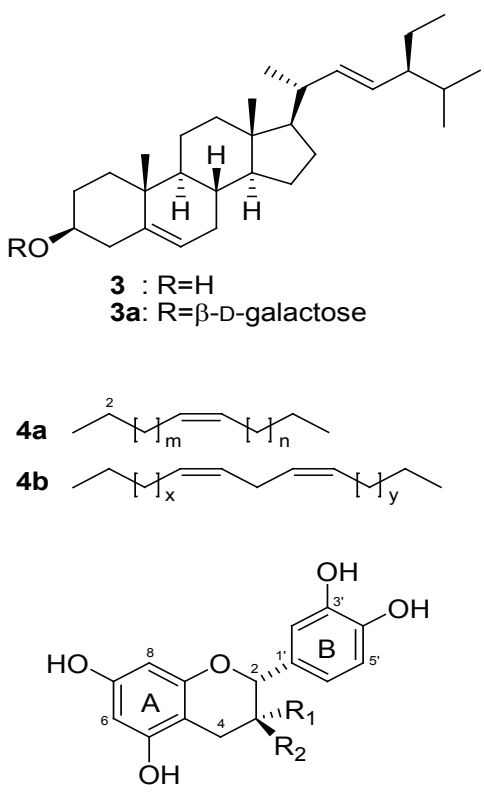

7: $\mathrm{R}_{1}=\mathrm{H} ; \mathrm{R}_{2}=\mathrm{OH}$ 8: $\mathrm{R}_{1}=\mathrm{OH} ; \mathrm{R}_{2}=\mathrm{H}$ 
Todas as substâncias isoladas são comuns em outras espécies, portanto suas estruturas foram identificadas por comparação dos dados de RMN ${ }^{1} \mathrm{H}$ e ${ }^{13} \mathrm{C}$ obtidos com os relatados na literatura.

A mistura de estigmasterol e sitosterol foi evidenciada no espectro de RMN ${ }^{1} \mathrm{H}$ pelos sinais em $\delta$ $3,54(m, \mathrm{H}-3)$ e $5,36(d l, J=2 \mathrm{~Hz}, \mathrm{H}-6)$ característicos de esteróis $\Delta^{5}$, sendo confirmado pelos sinais em $\delta 71,8$ (C-3), 140,8 (C-5) e 121,7 (C-6) do espectro de RMN ${ }^{13} \mathrm{C}$. A presença do estigmasterol na mistura foi definida pelos sinais de menor intensidade em $\delta 5,00$ e $5,19(d d, J=8$ e $15 \mathrm{~Hz}$ ) correspondentes a H-22 e H-23 da cadeia lateral e confirmado pelos sinais em $\delta 129,4$ e 138,3 referentes a C-23 e C-22 respectivamente (De-Eknamkul \& Potduang, 2003; Ayres et al., 2008). No espectro de RMN ${ }^{13} \mathrm{C}$ da mistura dos galactosídeos de sitosterol e estigmasterol e do 3-O-B-D-glicopiranosídeo do sitosterol foi observado desblindagem de C-3 $(\delta 79,6)$ em comparação aos esteroides livres $(\delta 71,8)$, além de sinais característicos da unidade de açúcar (Ahmed et al., 1992; Matilda et al., 1996). Os ésteres de sitosterol apresentaram espectros de RMN nos quais os sinais de $\mathrm{H}-3(\delta 4,60)$ e C-3 $(\delta 73,7)$ encontraramse na região de maior desblindagem em comparação aos do sitosterol (Parmar et al., 1998). Após hidrólise dos ésteres de sitosterol, a confirmação da estrutura da unidade esteroidal foi determinada por RMN. Os ácidos graxos foram metilados e os ésteres metílicos correspondentes foram analisados por $\mathrm{CG} / \mathrm{EM}$ onde o cromatograma de íons totais apresentou três picos com tempo de retenção de 23,$8 ; 27,5$ e $27,6 \mathrm{~min}$, correspondentes ao palmitato $(38,4 \%)$, linoleato $(8,7 \%)$ e oleato $(52,9 \%)$ de metila respectivamente.

A mistura dos ácidos anacárdicos foi identificada pela presença no espectro de RMN ${ }^{1} \mathrm{H}$ de três sinais em $\delta$ $6,83(d l, J=8,0 \mathrm{~Hz}), 6,74(d l, J=7,0 \mathrm{~Hz})$ e $7,33(t, J=7,0$ $\mathrm{Hz}$ ), característicos de anel aromático 1,2,3-trissubstituído juntamente com um tripleto em $\delta 2,94(J=7,7 \mathrm{~Hz})$ referente ao grupo metilênico $\alpha$ ao anel. Adicionalmente o espectro apresentou, entre outros, um multipleto situado na região de $\delta 5,32-5,38$ referente a hidrogênios olefínicos e dois outros multipletos em $\delta 2,01$ e 2,75, característicos de grupos metilênicos alílico e bis-alílico respectivamente, confirmando a presença de insaturação na cadeia lateral. A comparação das áreas integradas destes sinais sugeriu a presença de ácidos anacárdicos monoeno e dieno, sendo confirmado no espectro de $\mathrm{RMN}{ }^{13} \mathrm{C}$ pela presença de seis sinais de carbonos metínicos olefínicos $(\delta 128,0 ; 128,3$; $129,9 ; 130,0 ; 130,2$ e 130,3$)$. A atribuição dos sinais de carbono do anel aromático foi feita aplicando-se o princípio da aditividade do substituinte (Silverstein et al., 2006), que mostrou uma perfeita correlação com os dados obtidos: $\delta$ 110,6 (C-1), 163,6 (C-2), 115,8 (C-3), 135, 2 (C-4), 122,7 (C-5), 147,7 (C-6), 175,5 (C= O), 36,5 (C-1'). Todos os dados de $\mathrm{RMN}{ }^{1} \mathrm{H}$ e ${ }^{13} \mathrm{C}$ observados foram consistentes com os relatados por Itokawa et al. (1987).

A mistura de $\beta$-amirina e lupeol apresentou espectro de RMN ${ }^{1} \mathrm{H}$ com um duplo dupleto em $\delta 3,21$ $(J=4,3$ e $11,6 \mathrm{~Hz}, \mathrm{H}-3)$, característico de triterpenoides $3 \beta-\mathrm{OH}$, sendo confirmado pela presença do sinal em $\delta 79,0$ (C-3) no espectro de RMN ${ }^{13} \mathrm{C}$. A ocorrência do lupeol e $\beta$-amirina foi determinada de acordo com a metodologia descrita na literatura para análise de mistura de triterpenoides, devido a existência de dois pares de sinais em $\delta 109,5 / 151,2 ; 121,9 / 145,4$ característicos dos carbonos olefínicos nos esqueletos triterpênicos lupano e oleanano, respectivamente (Olea \& Roque, 1990; Mahato \& Kundu, 1994).

$\mathrm{O}$ espectro de $\mathrm{RMN}{ }^{1} \mathrm{H}$ da mistura de $7+8$ apresentou sinais em $\delta 2,49(d d, J=7,9$ e $16,0 \mathrm{~Hz}), 2,84$ $(d d, J=5,1$ e $16,0 \mathrm{~Hz}) 3,96(\mathrm{~m})$ e $4,55(d, J=7,5 \mathrm{~Hz})$ indicativos de flavan-3-ol e atribuídos respectivamente a $\mathrm{H}-4_{a x}, \mathrm{H}-4_{e q}, \mathrm{H}-3$ e H-2 da catequina (7). Adicionalmente apresentou dois dupletos em $\delta 5,84(J=2,1 \mathrm{~Hz})$ e $5,92(J=$ $2,1 \mathrm{~Hz}$ ) atribuídos a H-8 e H-6 do anel A, juntamente com sinais referentes ao anel B: $\delta 6,82\left(s l, \mathrm{H}^{\prime} 2^{\prime}\right), 6,75(d, J=$ $\left.8,1 \mathrm{~Hz}, \mathrm{H}-5^{\prime}\right)$ e $6,70(\mathrm{dl}, J=8,1 \mathrm{~Hz}, \mathrm{H}-6$ '). A epicatequina foi evidenciada pela presença do simpleto largo em $\delta 4,64$ (Shen et al., 1993) referente a H-4. O espectro de RMN ${ }^{13} \mathrm{C}$ apresentou dezesseis sinais, sendo quatorze referentes aos dois flavanóis, destes $\delta$ 28,5 (C-4) e 68,6 (C-3), juntamente com os sinais em $\delta 82,8$ e 76,9, atribuídos a C-2 da catequina (7) e epicatequina (8), respectivamente confirmaram a existência das duas substâncias. Todos os dados de RMN observados foram consistentes com os relatados na literatura (Shen et al., 1993; Jung et al., 2008).

\section{Avaliação do conteúdo de compostos fenólicos e atividade antioxidante}

Os compostos fenólicos de plantas se enquadram em diversas categorias, tais como: fenóis simples, ácidos fenólicos (derivados de ácidos benzóico e cinâmico), cumarinas, flavonoides, estilbenos, taninos condensados e hidrolisáveis, lignanas e ligninas (Sousa et al., 2007). Os compostos fenólicos naturais têm recebido muita atenção nos últimos anos, sobretudo por inibirem a peroxidação lipídica e a lipooxigenase in vitro (Haslam, 1996). Um dos métodos mais utilizados para determinação dos fenóis totais utiliza o reagente de Folin-Ciocalteu que consiste de mistura dos ácidos fosfomolibídico e fosfotunguístico, no qual o molibdênio e o tungstênio encontram-se no estado de oxidação $6+$ porém, em presença de certos agentes redutores, como os compostos fenólicos, formam-se o molibdênio azul e o tungstênio azul, nos quais a média do estado de oxidação dos metais está entre 5 e 6 e cuja coloração permite a determinação da concentração das substâncias redutoras (Naczk \& Shahidi, 2004; Ikawa et al., 2003).

A avaliação dos extratos de $A$. occidentale pelo método de Folin-Ciocalteu mostrou que a casca do caule é mais rica em compostos fenólicos do que o tegumento 
da castanha in natura apresentando $345,16 \pm 16,24$ e $185,44 \pm 12,04 \mathrm{mg}$ de equivalentes de ácido gálico, respectivamente.

O DPPH é um radical livre estável que tem sido amplamente usado para avaliar a atividade antioxidante de extratos e substâncias puras (Sousa et al., 2007; Jung et al., 2008). O efeito de antioxidantes no sequestro do DPPH é devido a capacidade doadora de hidrogênio destas substâncias as quais são geralmente compostos fenólicos. No presente estudo foi investigado o potencial dos extratos EtOH de cascas do caule e do tegumento da castanha in natura de $A$. occidentalle para sequestrar o radical DPPH. Os resultados obtidos, expressos por meio da porcentagem de atividade antioxidante nas concentrações de 25 a 250 $\mu \mathrm{g} / \mathrm{mL}$ são apresentados na Figura 1. Os dois extratos apresentaram percentual de atividade antioxidante dependente da concentração, porém a partir de $100 \mu \mathrm{g} /$ $\mathrm{mL}$ o extrato das cascas do caule não apresentou variação significativa, sendo mais ativo, em todas as concentrações testadas, do que o extrato do tegumento e do que o antioxidante sintético BHT (butil-hidroxitolueno) usado como controle positivo.

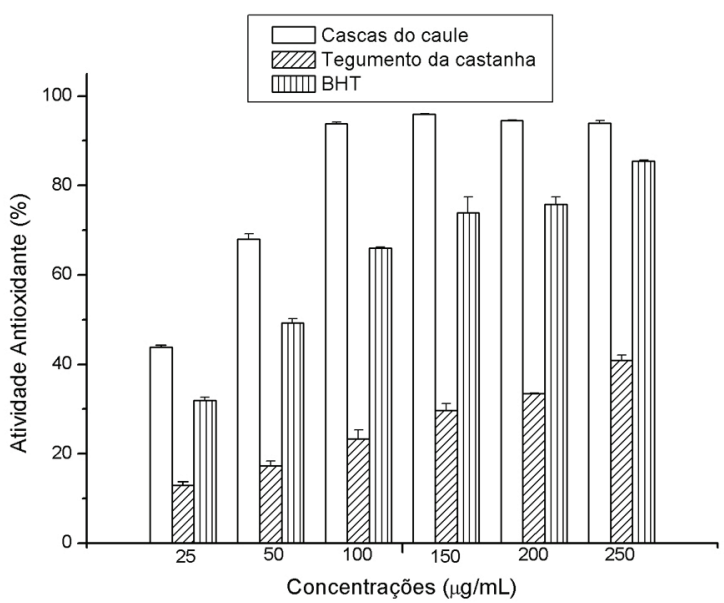

Figura 1. Percentual de atividade antioxidante de extratos EtOH de $A$. occidentale e do controle positivo BHT.

\section{CONCLUSÃO}

O fracionamento do extrato etanólico das cascas do caule de Anacardium occidentale L., Anacardiaceae resultou no isolamento de uma mistura de ácidos anacárdicos com cadeia lateral insaturada (monoeno e dieno), esteroides livres, glicosilados e esterificados com ácidos graxos. O extrato etanólico do tegumento da castanha de caju in natura apresentou os esteroides sitosterol e estigmasterol, os triterpenoides pentacíclicos lupeol e $\beta$-amirina e os flavanóis catequinas e epicatequina, isolados em mistura.

O lupeol, os ésteres e os glicosídios dos esteroides estão sendo relatados pela primeira vez em $A$. occidentale e, além disto, os ácidos anacárdicos ainda não haviam sido isolados de cascas do caule.

O extrato etanólico das cascas do caule apresentou maior conteúdo de compostos fenólicos, determinados pelo método de Folin-Ciocalteu, e maior potencial antioxidante no ensaio do radical DPPH, provavelmente decorrente da presença de ácidos anacárdicos e de taninos hidrolisáveis e não hidrolisáveis, obtidos abundantemente em cascas do caule de $A$. occidentale (Mota, 1982; Mota et al., 1985). Entretanto a atividade antioxidante do extrato do tegumento da castanha deve ser atribuída pelo menos em parte à presença da catequina e epicatequina, pois são substâncias que têm sido relatadas por possuir excelente efeito inibidor do radical DPPH. Estes resultados sugerem que os extratos EtOH, sobretudo o da casca do caule de $A$. occidentale poderá ser uma alternativa como aditivo em alimentos e preparações farmacêuticas e cosméticas em substituição aos antioxidantes sintéticos tóxicos.

\section{REFERÊNCIAS BIBLIOGRÁFICAS}

Agra MF, França PF, Barbosa-Filho JM 2007. Synopsis of the plants known as medicinal and poisonous in Northeast of Brazil. Rev Bras Farmacogn 17: 114-140.

Ahmed W, Ahmad Z, Malik A 1992. Stigmasteryl galactoside from Rhynchosia minima. Phytochemistry 31: 40384039.

Arya R, Babu V, Ilyas M 1989. Phytochemical examination of the leaves of Anacardium occidentale. J Indian Chem Soc 66: 67-68.

Ayres MCC, Brandão MS, Vieira-Júnior GM, Menor JCAS, Silva HB, Soares MJS, Chaves MH 2008. Atividade antibacteriana de plantas úteis e constituintes químicos da raiz de Copernicia prunifera. Rev Bras Farmacogn 18: 90-97.

Chaves MH, Barbosa AS, Moita Neto JM, Aued-Pimentel S, Lago JHG 2004. Caracterização química do óleo da amêndoa de Sterculia striata St. Hil. et Naud. Quim Nova 27: 404-408.

Correia SJ, David, JP, David JM 2006. Metabólitos secundários de espécies de Anacardiaceae. Quim Nova 6: 12871300.

De-Eknamkul W, Potduang B 2003. Biosynthesis of $\beta$-sitosterol and stigmasterol in Croton sublyratus proceeds via a mixed origin of isoprene units. Phytochemistry 62: 389398.

Dinda B, Chatterjee J, Banerjee J 1987. Sterol from Anacardium occidentale. J Indian Chem Soc LXIV: 647-648.

Gedam PH, Sampathkumaran PS 1986. Cashew nut shell liquid: extraction, chemistry and applications. Prog Org Coat 14: 115-157.

Grazzini R, Hesk D, Heininger E, Hildenbrandt G, Reddy CC, Cox-Foster D, Medford J, Craig R, Mumma RO 1991. Inhibition of lipoxygenase and prostaglandin endoperoxide synthase by anacardic acids. Biochem Biophys Res Commun 176: 775-780.

Haslam E 1996. Natural polyphenols (vegetable tannins) as drugs: possible modes of action. J Nat Prod 59: 205-215.

Himejima M, Kubo I 1991. Antibacterial agents from the cashew 
Anacardium occidentale (Anacardiaceae) nut shell oil. $J$ Agric Food Chem 39: 418-421.

Ikawa M, Schaper TD, Dollard CA, Sasner JJ 2003. Utilization of Folin-Ciocalteu phenol reagent for the detection of certain nitrogen compounds. J Agric Food Chem 51: 1811-1815.

Itokawa H, Totsuka N, Nakahara K, Takeya K, Lepoittevin J-P, Asakawa Y 1987. Antitumor principles from Ginkgo biloba L. Chem Pharm Bull 35: 3016-3020.

Jung MJ, Heo, S, Wang M-H 2008. Free radical scavenging and total phenolic contents from methanolic extracts of Ulmus davidiana. Food Chem 108: 482-487.

Kamath V, Rajini PS 2007. The efficacy of cashew nut (Anacardium occidentale L.) skin extract as a free radical scavenger. Food Chem 103: 428-433.

Kubo I, Komatzu S, Ochim M 1986. Molluscicides from the cashew Anacardium occidentale and their large-scale isolation. J Agric Food Chem 34: 970-973.

Kubo I, Kim M, Naya K, Komatzu S, Yamagiwa Y, Ohashi K, Sakamoto Y, Hirakawa S, Kamikawa T 1987. Prostaglandin synthetase inhibitors from the African medicinal plant Ozoroa mucronata. Chem Lett 16: 11011104.

Kubo I, Ochim M, Vieira PC, Komatsu S 1993. Antitumor agents from the cashew (Anacardium occidentale) apple juice. $J$ Agric Food Chem 41: 1012-1015.

Kubo I, Masuoka N, Ha TJ, Tsujimoto K 2006. Antioxidant activity of anacardic acids. Food Chem 99: 555-562.

Mahato SB, Kundu AP 1994. ${ }^{13} \mathrm{C}$ spectra of pentacyclic triterpenoids-a compilation and some salient features. Phytochemistry 37: 1517-1575.

Matilda AK, Rossi ME, Blumenthai EEA, Schuquel JTA, Malheiros A, Vidott GS 1996. Glucopyranosylsitosterol in species of Labiatae, Verbenaceae and Apocynaceae. Anais Assoc Bras Quim 43: 147-151.

Mota MLR 1982. Estudo antiinflamatório e análise química da casca do Anacardium occidentale $L$. João Pessoa, 184 p. Dissertação de Mestrado, Programa de Pós-Graduação em Produtos Naturais e Sintéticos Bioatiovos, Universidade Federal da Paraíba.

Mota MLR, Thomas G, Barbosa Filho JM 1985. Antiinflammatory actions of tannins isolated from the bark of Anacardium occidentale L. J Ethnopharmacol 13: 289300.

Mothé CG, Milfont Jr WN 1994. Aplicações do LCC (Líquido da Casca da Castanha de Caju). Rev Quim Ind 695: 15-19.

Moura D 2007. Castanha de caju proposta de preço mínimo safra 2006/2007. http://www.conab.gov.br/conabweb/ download/precos minimos/proposta de precos minimos_safra_2006_07_castanha_de_caju.pdf, acesso em fevereiro 2008

Murthy SSN, Anjaneyulu ASR, Row LR 1981. Occidentoside-a new biflavonoid glycoside from the nut shells of Anacardium occidentale Linn. Indian J Chem B 20: 150151.

Murthy SSN, Anjaneyulu ASR, Row LR, Pelter A, Ward S 1982. Chemical examination of Anacardium occidentaleisolation and structure determination of a novel biflavonoid-C-glycoside. Planta Med 45: 3-10.

Naczk M, Shahidi F 2004. Extraction and analysis of phenolics in food. J Chromatogr A: 95-111.

Olea RSG, Roque NF 1990. Analise de misturas de triterpenos por RMN de ${ }^{13}$ C. Quim Nova 13: 278-281.
Parmar VS, Jain SC, Gupta S, Talwar S, Rajwanshi VK, Kumar R, Azim A, Malhotra S, Kumar N, Jain R, Sharma NK, Tyagi OD, Lawrie SJ, Errington W, Howarth OW, Olsen CE, Singh SK, Wengel J 1998. Polyphenols and alkaloids from Piper species. Phytochemistry 49: 1069-1078.

Pio Corrêa M 1926. Dicionário de plantas Úteis do Brasil. Rio de Janeiro: Imprensa Nacional.

Rahman W, Ishratullah K, Wagner H, Seligmann O, Chari VM, Österdahl B-G 1978. Prunin-6"-O-p-coumarate, a new acylated flavanone glycoside from Anacardium occidentale. Phytochemistry 17: 1064-1065.

Shen C-C, Chang Y-S, Ho L-K 1993. Nuclear magnetic resonance studies of 5,7-dihydroxyflavonoids. Phytochemistry 34: 843-845.

Shobba SV, Krishnaswamy PR, Ravindranath B 1992. Phenolic lipid composition during development of cashew. Phytochemistry 31: 2295-2297.

Silva HR, Silva CCM, Caland Neto LB, Lopes JAD, Citó AMGL, Chaves MH 2007. Constituintes químicos das cascas do caule de Cenostigma macrophyllum: ocorrência de colesterol. Quim Nova 30: 1877-1881.

Silverstein RM, Webster FX, Kiemle DJ 2006. Identificação espectrométrica de compostos orgânicos. Rio de Janeiro: LTC.

Sousa CMM, Silva HR, Vieira-Jr GM, Ayres MCC, Costa CLS, Araújo DS, Cavalcante LCD, Barros EDS, Araújo PBM, Brandão MS, Chaves MH 2007. Fenóis totais e atividade antioxidante de cinco plantas medicinais. Quim Nova 30: 351-355.

Subramanian SS, Joseph KJ, Nair AGR 1969. Polyphenols of Anacardium occidentale. Phytochemistry 8: 673-673.

Subramanian SS, Nair AGR 1969. Catechins from cashewnut testa. Curr Sci India 38: 494-495.

Toschi TG, Caboni MF, Penazzi G, Lercker G, Capella P 1993. A study on cashelw nut oil composition. $\mathrm{J} \mathrm{Am} \mathrm{Oil} \mathrm{Chem}$ Soc 70: 1017-1020.

Tychopoulos V, Tyman JHP 1990. Long chain phenols-the thermal and oxidative deterioration of phenolic lipids from the cashew (Anacardium occidentale) nut shell. $J$ Sci Food Agric 52: 71-83.

Tyman JHP, Johnson RA., Muir M, Rokhgar R 1989. The extraction of natural cashew nut-shell liquid from the cashew nut (Anacardium occidentale). J Am Oil Chem Soc 66: 553-557. 